DiRECTEUR DE LA PUBLICATION / PUBLICATION DIRECTOR: Bruno David

Président du Muséum national d'Histoire naturelle

RÉDACTRICE EN CHEF / EDITOR-IN-CHIEF: Joséphine Lesur

RÉDACTRICE / EDITOR: Christine Lefèvre

RESPONSABLE dES ACTUALITÉS SCIENTIFIQUES / RESPONSIBLE FOR SCIENTIFIC NEWS: Rémi Berthon

AssisTANTE DE RÉDACTION / ASSISTANT EDITOR: Emmanuelle Rocklin (anthropo@mnhn.fr)

MISE EN PAGE / PAGE LAYOUT: Emmanuelle Rocklin, Inist-CNRS

COMITÉ SCIENTIFIQUE / SCIENTIFIC BOARD:

Louis Chaix (Muséum d'Histoire naturelle, Genève, Suisse)

Jean-Pierre Digard (CNRS, Ivry-sur-Seine, France)

Allowen Evin (Muséum national d'Histoire naturelle, Paris, France)

Bernard Faye (Cirad, Montpellier, France)

Carole Ferret (Laboratoire d'Anthropologie Sociale, Paris, France)

Giacomo Giacobini (Università di Torino, Turin, Italie)

Lionel Gourichon (Université de Nice, Nice, France)

Véronique Laroulandie (CNRS, Université de Bordeaux 1, France)

Stavros Lazaris (Orient \& Méditerranée, Collège de France - CNRS - Sorbonne Université, Paris, France)

Nicolas Lescureux (Centre d'Écologie fonctionnelle et évolutive, Montpellier, France)

Marco Masseti (University of Florence, Italy)

Georges Métailié (Muséum national d'Histoire naturelle, Paris, France)

Diego Moreno (Università di Genova, Gènes, Italie)

François Moutou (Boulogne-Billancourt, France)

Marcel Otte (Université de Liège, Liège, Belgique)

Joris Peters (Universität München, Munich, Allemagne)

François Poplin (Muséum national d'Histoire naturelle, Paris, France)

Jean Trinquier (École Normale Supérieure, Paris, France)

Baudouin Van Den Abeele (Université Catholique de Louvain, Louvain, Belgique)

Christophe Vendries (Université de Rennes 2, Rennes, France)

Denis Vialou (Muséum national d'Histoire naturelle, Paris, France)

Jean-Denis Vigne (Muséum national d'Histoire naturelle, Paris, France)

Arnaud Zucker (Université de Nice, Nice, France)

COUVERTURE / COVER:

Bois de cerf dans la coupe stratigraphique nord-sud du secteur 1, château de Talmon (crédit photo T. Bethus) / Deer antlers in the north-south stratigraphic section of sector 1, Talmon Castle (photo credit T. Bethus).

Anthropozoologica est indexé dans / Anthropozoologica is indexed in:

- Social Sciences Citation Index

- Arts \& Humanities Citation Index

- Current Contents - Social \& Behavioral Sciences

- Current Contents - Arts \& Humanities

- Zoological Record

- BIOSIS Previews

- Initial list de l'European Science Foundation (ESF)

- Norwegian Social Science Data Services (NSD)

- Research Bible

Anthropozoologica est distribué en version électronique par / Anthropozoologica is distributed electronically by:

- BioOne ${ }^{\circledR}$ (http://www.bioone.org)

Anthropozoologica est une revue en flux continu publiée par les Publications scientifiques du Muséum, Paris, avec le soutien du CNRS.

Anthropozoologica is a fast track journal published by the Museum Science Press, Paris, with the support of the CNRS.

Les Publications scientifiques du Muséum publient aussi / The Museum Science Press also publish:

Adansonia, Zoosystema, Geodiversitas, European Journal of Taxonomy, Naturae, Cryptogamie sous-sections Algologie, Bryologie, Mycologie.

Diffusion - Publications scientifiques Muséum national d'Histoire naturelle

CP 41 - 57 rue Cuvier F-75231 Paris cedex 05 (France)

Tél. : 33 (0)1407948 05 / Fax: 33 (0)1 40793840

diff.pub@mnhn.fr / http://sciencepress.mnhn.fr

(C) Publications scientifiques du Muséum national d'Histoire naturelle, Paris, 2020

ISSN (imprimé / print): 0761-3032 / ISSN (électronique / electronic): 2107-08817 


\title{
Des bestes médiévales aux espèces linnéennes: autour de la classification de quelques espèces en contexte castral au Moyen Âge
}

\author{
Aurélia BORVON \\ Archéologie et sciences de l'Antiquité (ArScAn), Équipe Archéologies environnementales, \\ CNRS, Université Paris 1 Panthéon Sorbonne, Université Paris Ouest Nanterre la Défense, \\ Ministère de la Culture, MSH Mondes, \\ 21 allée de I'Université, F-92023 Nanterre Cedex (France) \\ et Laboratoire d'Anatomie Comparée, École nationale vétérinaire, agroalimentaire \\ et de l'Alimentation (Oniris), Ministère de l'Agriculture et de I'Alimentation, \\ 101 Route de Gachet, F-44300 Nantes (France) \\ et Groupe d'Études Remodelage osseux et bioMatériaux (GEROM), Université d'Angers, \\ Institut de Biologie en Santé, Iris, 4 Rue Larrey, CHU, F-49933 Angers (France). \\ aureliaborvon@gmail.com \\ Charles VIAUT \\ Centre d'études supérieures de civilisation médiévale, Université de Poitiers, CNRS, \\ Maison des Sciences de I'Homme et de la Société, Bâtiment A5, Bureau 116, \\ 5 rue Théodore Lefebvre, TSA 2110, F-86073 Poitiers Cedex 9 (France) \\ charles.viaut@univ-poitiers.fr
}

Soumis le 14 octobre 2019 | Accepté le 8 avril 2020 | Publié le 17 juillet 2020

MOTS CLÉS

Archéologie castrale, archéozoologie, histoire de l'alimentation,
Borvon A. \& Viaut C. 2020. - Des bestes médiévales aux espèces linnéennes: autour de la classification de quelques espèces en contexte castral au Moyen Âge, in Brémont A., Boudes Y., Thuault S. \& Ben Saad M. (éds), Appréhender les catégories zoologiques dans les sociétés du passé. Anthropozoologica 55 (10): 145-157. https://doi.org/10.5252/ anthropozoologica2020v55a10. http://anthropozoologica.com/55/10

\section{RÉSUMÉ}

Les recherches menées actuellement sur plusieurs sites castraux médiévaux de l'Ouest de la France, notamment sur le château de Talmont (Vendée) et le château de Thouars (Deux-Sèvres), sont inscrites dans une démarche interdisciplinaire conjuguant les données issues de l'archéozoologie et celles des sources écrites, ce qui permet de mettre en valeur la grande variété des espèces consommées ou utilisées par l'homme. Les animaux sauvages, consommés ou non, ont fait l'objet d'une attention particulière. L'étude des pratiques alimentaires sur ces sites s'accompagne ainsi de l'étude des textes relatifs aux animaux, à leur élevage et à leur consommation, qu'il s'agisse de comptes ou d'actes. Ces documents, qui permettent dans bien des cas d'affiner la compréhension des contextes archéologiques et du fonctionnement économique et social des sites castraux, ne s'accordent pas systématiquement sur un point précis: la considération des différentes espèces. Si l'archéozoologie classe les restes osseux selon les canons de la biologie actuelle, les documents médiévaux de la pratique donnent à voir un discours qui s'éloigne considérablement de ces logiques modernes. La comparaison de ces deux discours à l'échelle de deux sites permet de mettre en lumière les différences d'approche et de questionner les interprétations actuelles des nomenclatures du monde animal au Moyen Âge. 
KEY WORDS

Castle archaeology, archaeozoology,

food history, cultural history.

\begin{abstract}
From medieval beasts to linnean species: around the classification of some species in castral context in the Middle Ages.

The research currently conducted on several medieval castle sites in western France, in particular Talmont Castle (Vendée, France) and Thouars Castle (Deux-Sèvres, France), is being carried out in an interdisciplinary manner: the study of bone remains using archaeozoological methods makes it possible to highlight the wide variety of species consumed or used by humans for whatever reason. Wild animals, whether consumed or not, have thus received special attention. The study of feeding practices on these sites is also accompanied by a study of texts relating to animals, their breeding and consumption, whether they are accounts or documents of practice. These documentary sources, which in many cases make it possible to refine the understanding of the archaeological contexts and the economic and social functioning of castle sites, do not lend themselves to an exact comparison on one point: the consideration of the different species. If archaeozoology classifies bone remains according to the canons of contemporary biology, the documents of medieval practice show a discourse that is specific to them and that sometimes differs considerably from the logic of current science. The comparison of these two discourses at the site level allows us to highlight the differences in approach and to question our current interpretations of representations of the animal world in the Middle Ages.
\end{abstract}

\section{INTRODUCTION}

Les programmes de recherche menés depuis plusieurs années sur certains sites castraux de l'Ouest de la France abordent désormais systématiquement, entre autres aspects, les questions d'alimentation et de consommation : ainsi sur les sites de Suscinio (Morbihan) (Borvon 2017a; Vincent 2017; Vincent et al. 2018) et de Talmont (Vendée) (Béthus \& Prouteau 2014; Béthus 2016; Borvon 2016, 2017b; Viaut 2018a, 2018b; Borvon \& Viaut 2018). Les analyses peuvent y être menées de manière satisfaisante en raison de l'abondance des vestiges fauniques sur ces sites élitaires et sur lesquels les analyses archéozoologiques sont toujours en cours. Les macrorestes sont ramassés tant manuellement lors de la fouille que via un tri des sédiments préalablement prélevés et tamisés (pour une référence générale sur ces aspects méthodologiques, voir Horard-Herbin \& Vigne 2005). L'une des étapes primordiales des analyses archéozoologiques est l'établissement de la liste des espèces présentes sur le site. L'identification de ces dernières repose sur l'anatomie comparée et le principe d'actualisme. Il n'est cependant pas toujours possible d'atteindre le rang de l'espèce, et l'emploi du mot taxon - terme qui désigne toute unité systématique, indépendamment de son rang (famille, genre, espèce, etc.; Lecointre \& Le Guyader 2001) - est souvent bien pratique. En plus de son nom vernaculaire, une espèce est désignée par une terminologie latine actuellement de rigueur pour les nommer: la nomenclature dite binominale ou "linnéenne", du nom de celui qui en a généralisé l'usage au XVIIIe siècle, Carl von Linné. Le classement des espèces est, quant à lui, réalisé suivant la classification actuelle du vivant, dite phylogénétique, dont le cadre repose sur la théorie de l'évolution (Lecointre \& Leguyader 2001). Quelques «arrangements» avec cette classification sont néanmoins parfois opérés, notamment dans le cas des "poissons", groupe invalide à l'heure actuelle. Le terme continue pourtant à être utilisé pour faciliter la compréhension des analyses.
D'autres documents peuvent cependant être exploités dans le cadre des travaux sur l'alimentation et la consommation en contexte castral, à savoir les sources écrites médiévales concernant ces sites. Il n'est pas question ici de manuscrits d'œuvres littéraires ou encyclopédiques, beaucoup mises à contribution dans les travaux généraux sur l'alimentation, mais des documents de la pratique enregistrant la gestion quotidienne, le plus souvent annuelle, parfois même journalière des domaines seigneuriaux (Chanson 2007; Contamine 2011). Il convient de distinguer au sein de cet ensemble les comptes alimentaires, vaste catégorie désignant les documents consignant les dépenses de bouche au sein d'un hôtel seigneurial. Parmi eux peuvent être distingués, d'une part, les comptes journaliers plus ou moins bien tenus des petites seigneuries, regroupant des dépenses de tous ordres, pas seulement liées à l'alimentation, et d'autre part, les comptes strictement organisés en offices de bouche des grands hôtels princiers, royaux, épiscopaux ou pontificaux (Laurioux \& Moirez 2008). Dans l'espace francophone médiéval, les comptes des hôtels sont rarement conservés avant le XIIII siècle, sauf en Angleterre, notamment ceux de l'hôtel royal et des manors (Woolgar 1992), issus des petites seigneuries de l'île. Sur le continent, ces comptes d'hôtel sont encore particulièrement rares au XIVe siècle, à l'exception de ceux du roi et de certains grands princes, tels les ducs de Bretagne et de Bourgogne ou encore les comtes de Savoie (Morel 2006, 2008; Laurioux \& Moirez 2008; Moirez 2009; Abbott 2012; Casset 2018). La répartition de ces comptes au sein des fonds d'archives est largement tributaire, non seulement de la répartition géographique des pouvoirs à la fin du Moyen Âge, mais aussi de la diffusion des usages de l'écrit au sein des différentes strates de l'aristocratie, sans oublier les hasards de la conservation documentaire, qui empêchent alors toute généralisation à l'échelle du territoire actuel. Nous possédons en revanche beaucoup de comptes d'hôtels du XVe siècle issus des grands fonds d'archives seigneuriaux, de Bourgogne, de Bretagne ou encore de Thouars 


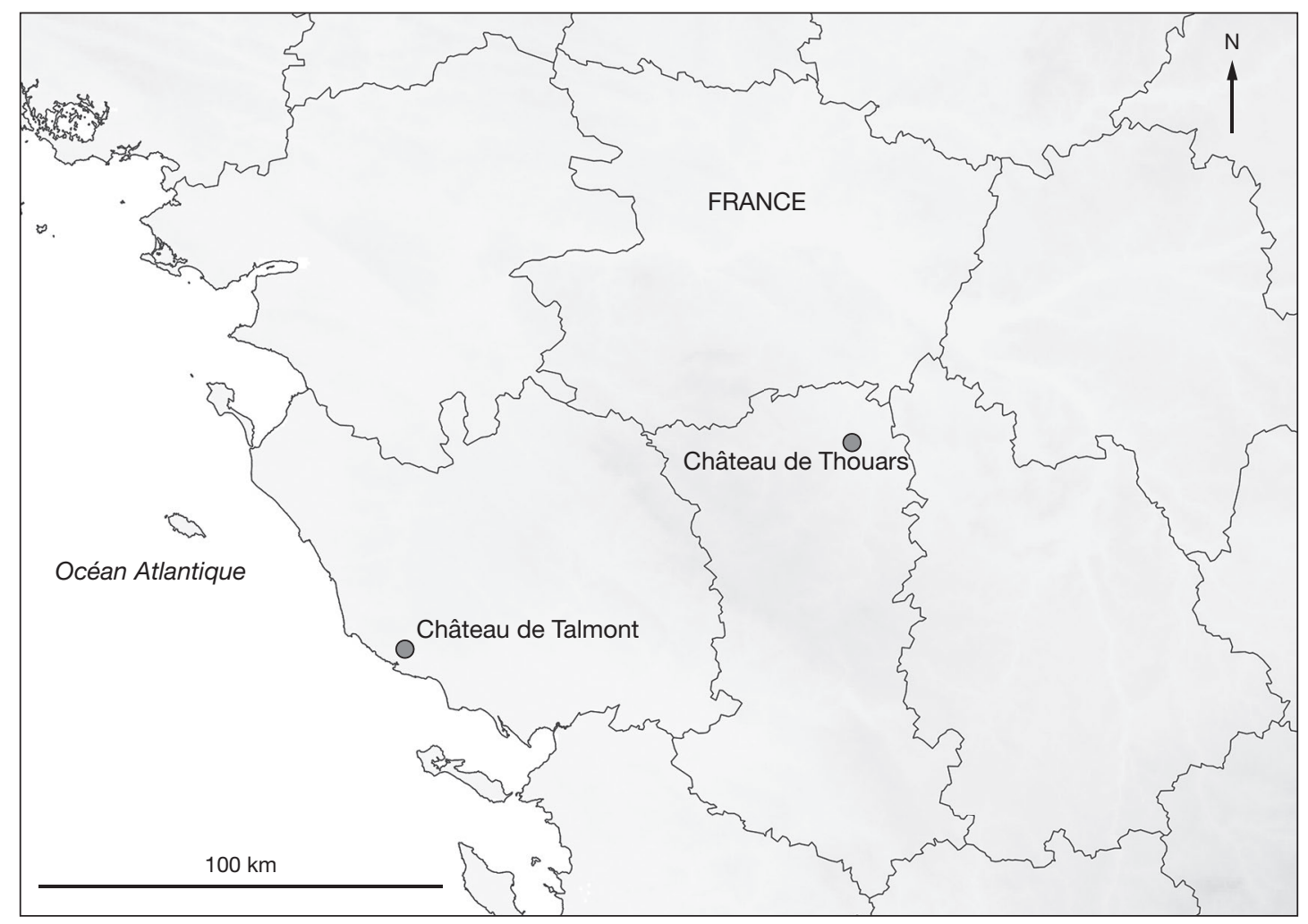

FIG. 1. - Localisation du château de Talmont et du château de Thouars (cartographie C. Viaut).

(Samaran \& Courteault 1930). Néanmoins, ceux émanant d'une aristocratie de rang plus modeste sont de plus en plus nombreux, notamment ceux des seigneurs châtelains de rang intermédiaire, voire de petits hobereaux (Charbonnier 1971; Casset 2017; Noël 2017). Parmi les hôtels seigneuriaux renseignés par les comptes dans la première moitié du XVe siècle, celui de Louis d'Amboise (vicomte de Thouars de 1422 à 1469), dont la consommation et l'approvisionnement en son château de Thouars sont précisément documentés, se distingue. Beaucoup de documents concernent également l'alimentation des vicomtes de Thouars au château de Talmont. Il s'est agi alors de conduire une approche interdisciplinaire à partir de corpus documentaires divers mettant en œuvre des méthodologies différentes: les investigations archéologiques conduites sur ce dernier site, les résultats des études archéozoologiques menées sur les niveaux datés de la fin du Moyen Âge (Borvon 2016, 2017b; Borvon \& Viaut 2018), contemporains des visites du vicomte ou de ses successeurs directs (Viaut 2018a, 2018b), ont été confrontées aux données des comptes de bouche de l'hôtel du vicomte. La mise en perspective des résultats de ces deux types de sources a rapidement révélé un décalage, parfois important, entre la manière dont sont distinguées les espèces animales retrouvées sur les sites castraux, décrites selon les codes de la biologie actuelle, et les discours qu'en ont laissé les occupants de ces sites dans les documents de gestion. Nous présenterons ici les deux sites, avant de comparer différents aspects des nomenclatures médiévales et actuelles des espèces animales en contexte castral.

\section{DEUX SITES LIÉS PAR L'HISTOIRE: \\ TALMONT (VENDÉE) ET THOUARS (DEUX- SĖVRES). PRÉSENTATION DU CONTEXTE ARCHÉOLOGIQUE ET HISTORIQUE DE LA DOCUMENTATION.}

Le site de Talmont, situé sur la commune de Talmont SaintHilaire, dans le sud-ouest du département de la Vendée (Fig. 1), a fait l'objet de recherches archéologiques et historiques entreprises entre 2003 et 2018 (responsable d'opération: T. Béthus) sous l'égide de l'Inrap Grand Ouest, puis du Conseil départemental de la Vendée et du Centre d'études supérieures de civilisation médiévale (CESCM) de l'Université de Poitiers. La chronologie de la construction et la nature de l'occupation du site ont pu être précisées sur une durée de plus de six siècles: de la fin du $\mathrm{X}^{\mathrm{e}}$ au début du XVIIe siècle. Comportant un monastère ou prieuré et une occupation annexe encore peu connue sur un promontoire surplombant la confluence de deux petits fleuves côtiers, le site connaît un fort développement à partir du début du XIe siècle, avec sa mise en défense par une enceinte ovalaire de pierre englobant quasiment toute la surface du promontoire et protégeant un bâtiment constitué d'une grande salle et d'espaces privatifs au nord-ouest de l'enceinte. Il s'agit probablement, d'après la documentation historique, d'une fondation des comtes de Poitiers, cherchant alors à étendre leur influence en direction du littoral. Une tour maîtresse attenante à la grande salle est érigée entre la seconde moitié du XIe et le début du XIIe siècle. Entre la fin du XIIe et le début du XIIIe siècle, les 
financements de la royauté Plantagenêt permettent à Savary de Mauléon, seigneur de Talmont, la construction d'un imposant programme de fortifications comprenant une tour-éperon et un mur-bouclier accolés à la tour maîtresse, une seconde enceinte interne et une enceinte externe englobant le site tout entier (Fig. 2). Ainsi, jusqu'au premier tiers du XIIIe siècle, le site castral de Talmont constitue un lieu de pouvoir majeur et un enjeu stratégique d'importance dans le contexte de l'expansion de la royauté capétienne au sud de la Loire (Béthus \& Prouteau 2014). À partir de la seconde moitié du XIIIe siècle, le château et la seigneurie de Talmont intègrent les vastes domaines des vicomtes de Thouars. Le site perd son potentiel stratégique mais garde tout son prestige de siège de grande seigneurie et de résidence palatiale, dans laquelle les vicomtes (du XIVe et du XVe siècle) effectuent des séjours réguliers pour la fête ou la chasse. La grande salle seigneuriale au nord continue à être occupée et réaménagée jusqu'au XVe siècle, époque à laquelle elle est finalement abandonnée. Les niveaux archéologiques de ce secteur ont livré des vestiges fauniques datés du XIe au $\mathrm{XV}$ siècle, relativement abondants dans les niveaux les plus tardifs. Pour ces dernières périodes, la mise en perspective de ces données avec les quelques documents d'archives conservés apporte l'éclairage le plus intéressant, permettant de caractériser l'alimentation dans ces ensembles castraux à la fin du Moyen Âge (Béthus 2016; Viaut 2018a). Par ailleurs, la position de Talmont au sein de l'ensemble des domaines des vicomtes de Thouars et son statut de résidence régulière pour les vicomtes permet des comparaisons avec un autre site, mieux pourvu en termes archivistiques: le château de Thouars (Fig. 1).

Siège du pouvoir des vicomtes de Thouars depuis le $\mathrm{Xe}$ siècle au moins (Jeanneau 2013), le château de Thouars est encore, au $\mathrm{XVe}$ siècle, le cour d'un vaste ensemble de seigneuries de l'ouest du royaume, tenues par les vicomtes et par leurs très nombreux vassaux. La reconstruction totale du château au XVIIe siècle après l'érection de la vicomté en duché a fait disparaître en grande partie les bâtiments médiévaux en élévation, et le sous-sol n'y a pas encore fait l'objet d'investigations archéologiques qui permettraient la découverte de vestiges médiévaux. La gestion des domaines des vicomtes au château de Thouars a donné naissance à un très vaste ensemble archivistique fusionné dès la fin du XVe siècle avec les archives familiales des La Trémoïlle, héritiers de la vicomté de Thouars et de la seigneurie de Talmont en 1487 (Vissière 2010). Cet ensemble, constamment enrichi jusqu'au XIXe siècle, a constitué le chartrier de Thouars, plus grand fonds d'archives privées actuellement préservé en France, conservé aux Archives Nationales sous la cote 1AP (Archives nationales: $1 \mathrm{AP} / 556,1 \mathrm{AP} / 559)$. Au sein de cet ensemble sont conservées notamment une grande partie des archives des vicomtes de Thouars de la maison d'Amboise, qui régnèrent une bonne part du XVe siècle à Thouars et à Talmont. Les comptes de l'hôtel du vicomte Louis d'Amboise permettent ainsi d'approcher la consommation alimentaire jour par jour sur huit années, entre 1440 et 1469, malgré d'importantes lacunes. Correspondant probablement à ceux de l'office de cuisine, bien que cela n'y soit jamais précisé, ils constituent la principale source disponible sur l'alimentation à la fin du Moyen Âge à Thouars.
L'approche de l'alimentation seigneuriale à Thouars et Talmont à cette époque est ainsi semblable à un patient travail de couture entre des sources disjointes: les riches archives du château de Thouars d'une part, qui ne couvrent cependant que quelques années et ne peuvent pas être comparées aux sources matérielles sur place; les vestiges archéologiques et les quelques documents du château de Talmont d'autre part, où les visites et l'occupation des vicomtes sont largement attestées par les sources écrites à la fin du Moyen Âge. Les recherches historiques ont par ailleurs permis de mettre en évidence une importante circulation de produits alimentaires organisée par les seigneurs entre ces deux sites, notamment en direction de Thouars (Viaut 2018a, 2018b). Leurs liens historiques, ainsi que les datations solides des faits archéologiques comme des données archivistiques, nous convainquent de la pertinence de la comparaison entre ces deux sites en dépit des divergences documentaires signalées plus haut. La comparaison entre les nomenclatures animalières de l'archéozoologue et le vocabulaire du comptable médiéval ont permis de mettre en évidence d'intéressants phénomènes de divergences et de convergences à prendre en compte lors de la synthèse des données.

\section{DISCORDANCE ET CONVERGENCES ENTRE TERMINOLOGIE COMPTABLE MÉDIÉVALE ET NOMENCLATURE SCIENTIFIQUE ACTUELLE}

\section{LES APPROCHES QUANTITATIVES : COMPTAGE DES OS ET DÉNOMBREMENTS DANS LES TEXTES \\ La triade domestique, une prédominance dans les deux types de sources}

L'approche des espèces animales consommées en contexte castral passe nécessairement par une approche quantitative, qu'elle relève du nombre de restes et de leur masse en archéozoologie ou du nombre de mentions dans les sources écrites. Sur les sites archéologiques, la caractéristique majeure de l'alimentation, et ce depuis le Néolithique, est la domination d'un trio d'espèces domestiques tant en masse qu'en nombre de restes. Le Moyen Âge et les contextes castraux ne font généralement pas exception à cette règle, bien qu'il existe des variations temporelles et spatiales (e.g. AudoinRouzeau1986, 1995; Clavel 2001; Borvon 2012) dans les proportions des trois taxons concernés, à savoir le bœuf (Bos taurus L., 1758), le porc (Sus scrofa f. domesticus Erxleben, 1777) et les caprinés, famille qui comprend le mouton (Ovis aries L., 1758) et la chèvre (Capra hircus L., 1758), espèces parfois difficiles à distinguer ostéologiquement. Cette triade domine ainsi largement les assemblages talmondais, avec une proportion qui oscille entre $70 \%$ et $90 \%$ des restes suivant les contextes (Table 1). Jusqu'au XIVe siècle, le porc domine en nombre de restes (environ $50 \%$ des restes de la triade), bien qu'en quantité de viande consommée, ce soit le bœuf qui prenne la première place ${ }^{1}$. À partir du XIVe siècle (et ultérieurement, Borvon \& Viaut 2018), il semblerait que les

1. En utilisant la masse des restes par exemple. Pour une synthèse des différentes méthodes d'approche quantitative de l'alimentation carnée, ainsi que leurs conditions d'application, cf. Borvon 2012: 363 et suivantes. 


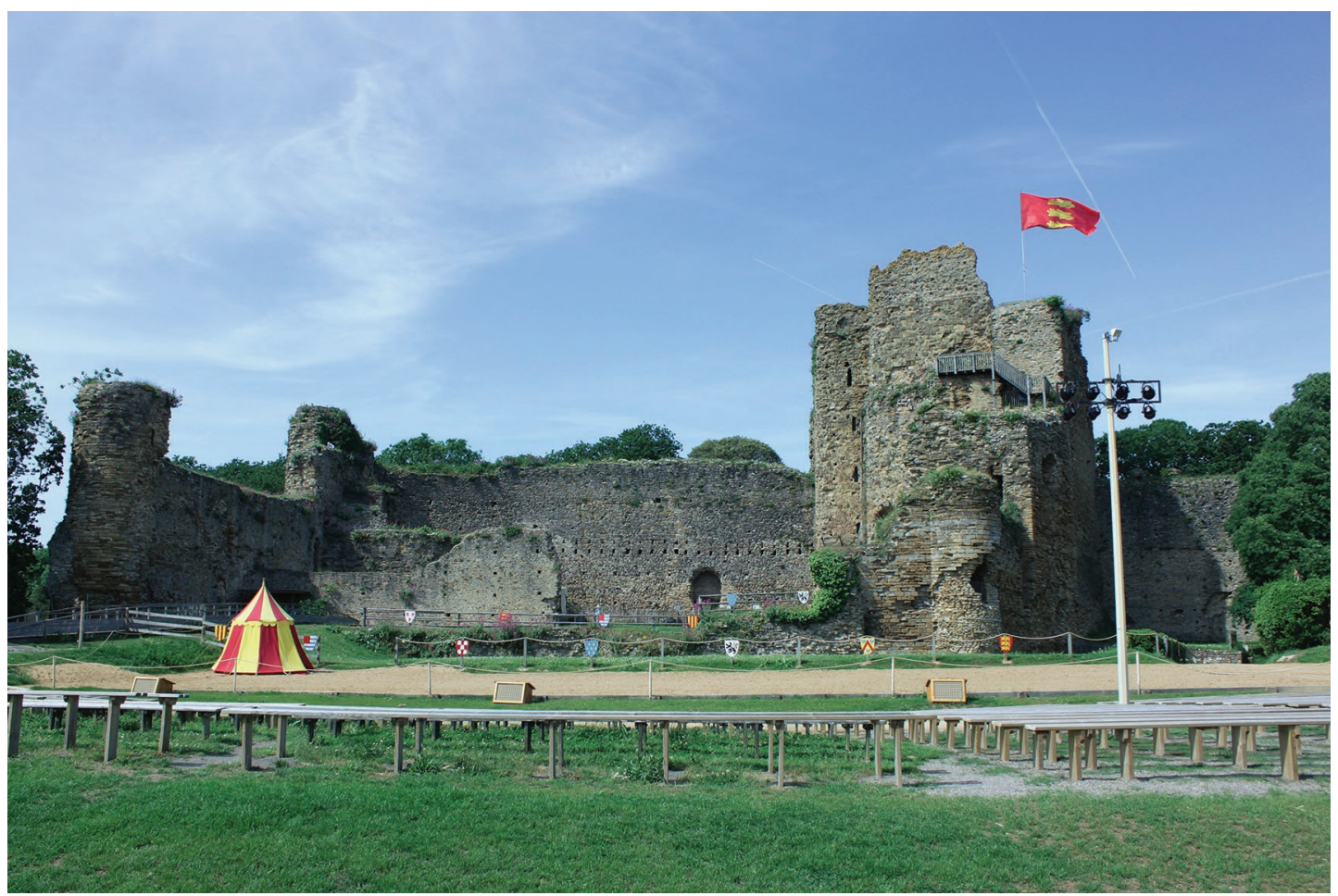

FIG. 2. - Courtine nord et tour maîtresse du château de Talmont (crédits photo C. Viaut).

caprinés prennent un peu plus d'importance en nombre de restes, sans que leur consommation ne devienne véritablement conséquente face au bœuf.

Cette même domination de la triade domestique peut également être observée dans les comptabilités. Les dépenses alimentaires consignées dans les comptes journaliers de l'année 1440 au château de Thouars sont à cet égard riches d'enseignements: entre le 22 mai et le 18 août 1440 (Archives nationales: 1AP/5591), les dépenses cumulées pour l'achat de viande de bœuf, de porc et de caprinés représentent $73 \%$ des dépenses en produits carnés, dont une majorité de ces derniers. Le bœuf et le porc, en nombre plus restreint, fournissent toutefois plus de viande (Fig. 3). L'hôtel du vicomte de Thouars achète durant cette période, du mouton, du porc, du bœuf mais également du chevreau, du veau ou encore du cochon de lait, et ce 95 fois sur l'intervalle chronologique considéré. Cette consommation de viande de qualité se retrouve également dans les niveaux archéologiques par la présence d'individus jeunes ${ }^{2}$ dans les assemblages osseux (Borvon 2017b). Par exemple, pour l'état 7 (XIII-XIVe siècle), deux agneaux et un chevreau sont recensés parmi les six individus dénombrés ${ }^{3}$; les autres, des moutons, ne sont pas non plus très vieux, avec un juvénile et deux subadultes. De la même manière, les porcs sont, toutes phases confondues, souvent des juvéniles (voire de tout jeunes subadultes) qui procurent de la viande fraîche de qualité.

2. Infantiles, juvéniles voire subadultes (concordances avec un âge précis pour la triade domestique dans Forest 1997) suivant les espèces. Les âges d'abattage sont déduits de l'observation des dents (éruptions et/ou usures dentaires) et/ou du squelette postcrânien (soudure des épiphyses).

3. Peu d'individus sont présents par phase chronologique, mais les grandes tendances paraissent globalement assez similaires.
Si les renseignements fournis par les textes ne permettent pas systématiquement d'accéder à des informations telles que le nombre d'animaux ou de pièces de viande acquis, la répartition des dépenses ou la fréquence des achats permettent d'évaluer le poids respectif des différentes espèces dans l'alimentation seigneuriale thouarsaise. La convergence avec les observations faites au château de Talmont est ici pertinente, tant en termes de répartition des espèces que d'âge à l'abattage. La présence d'animaux jeunes dans l'alimentation seigneuriale de la fin du Moyen Âge est aussi bien attestée dans les comptes thouarsais, notamment à propos des chevreaux, que dans les dépôts archéologiques talmondais. Cette observation rejoint bien d'autres constats faits au même moment sur d'autres ensembles seigneuriaux (Noël 2017).

\section{Animaux sauvages et chasse, sources écrites versus données archéozoologiques: l'exemple de l'état 9 du bâtiment nord du château de Talmont (fin XIVe-XVe siècle)}

$\mathrm{Si}$, quel que soit le paramètre de quantification pris en compte, la triade domestique domine en général largement les assemblages, à Talmont comme ailleurs, les espèces chassées peuvent parfois prendre l'avantage. C'est ainsi le cas des derniers niveaux d'occupation à Talmont (état 9: fin XIVe-XVe siècle). Au sein de ceux-ci, environ 800 restes de Mammifères sont identifiés, dont près de la moitié correspondent à des ossements d'espèces sauvages (Borvon \& Viaut 2018). Il s'agit essentiellement de restes de cerf (Cervus elaphus L., 1758), mais aussi de sanglier (Sus scrofa scrofa L., 1758). Cette proportion, souvent élevée sur les sites élitaires (synthèse dans Borvon 2012), correspond toutefois ici à un taux tout à fait exceptionnel, et semble 
TABLE 1. - Nombre de restes et masse des restes (en grammes) déterminés et indéterminés de mammifères, d'oiseaux et de poissons du château de Talmont pour les états 6 à 8 (XIIIe-XIVe siècle) du secteur 1 (bâtiment nord) (Borvon 2017b). Abréviations: MR, masse des restes; NR, nombre de restes.

\begin{tabular}{|c|c|c|c|c|c|c|c|c|c|c|c|}
\hline \multirow[b]{2}{*}{ Nom vernaculaire } & \multirow[b]{2}{*}{ Nomenclature linnéenne } & \multicolumn{2}{|c|}{ État 5 - XIIle } & \multicolumn{2}{|c|}{ État 6 - XIIIe-XIVe } & \multicolumn{2}{|c|}{ État $7-$ XIIIe-XIVe } & \multicolumn{2}{|c|}{ État $8-\mathrm{XIVe}$} & \multicolumn{2}{|c|}{ Total - XIIIe-XIVe } \\
\hline & & NR & MR & NR & MR & NR & MR & NR & MR & NR & MR \\
\hline \multicolumn{12}{|c|}{ Mammifères } \\
\hline Boeuf & $\begin{array}{l}\text { Bos taurus } \\
\text { Linnaeus, } 1758\end{array}$ & 11 & 496,1 & 49 & 2052,2 & 29 & 713,4 & 11 & 401,8 & 100 & 3663,5 \\
\hline Caprinés & Caprinae & 5 & 23,4 & 34 & 283,2 & 55 & 278,7 & 34 & 247,6 & 128 & 832,9 \\
\hline Porc & $\begin{array}{l}\text { Sus scrofa f. domesticus } \\
\text { Erxleben, } 1777\end{array}$ & 18 & 226,9 & 87 & 1182 & 76 & 420,1 & 20 & 185,5 & 201 & 2014,5 \\
\hline Sanglier & $\begin{array}{l}\text { Sus scrofa scrofa } \\
\text { Linnaeus, } 1758\end{array}$ & 4 & 44,8 & 3 & 612,6 & 6 & 88,7 & 5 & 103,7 & 18 & 849,8 \\
\hline Cerf & $\begin{array}{l}\text { Cervus elaphus } \\
\text { Linnaeus, } 1758\end{array}$ & 1 & 60,5 & 4 & 120,7 & 6 & 97,1 & 24 & 809,9 & 35 & 1088,2 \\
\hline Lièvre & $\begin{array}{l}\text { Lepus europaeus Pallas, } \\
1778\end{array}$ & 2 & 12,1 & 3 & 10,9 & 1 & 0,4 & 1 & 0,5 & 7 & 23,9 \\
\hline Lapin & $\begin{array}{l}\text { Oryctolagus cuniculus } \\
\text { (Linnaeus, 1758) }\end{array}$ & - & - & 5 & 5,7 & 13 & 12,4 & 2 & 3,6 & 20 & 21,7 \\
\hline Léporidés & Leporidae & 2 & 2,7 & 6 & 3,7 & 18 & 11 & 8 & 2 & 34 & 19,4 \\
\hline Chat & $\begin{array}{l}\text { Felis catus } \\
\quad \text { (Linnaeus, 1758) }\end{array}$ & - & - & - & - & 1 & 0,3 & - & - & 1 & 0,3 \\
\hline Rat noir & $\begin{array}{l}\text { Rattus rattus } \\
\quad \text { (Linnaeus, 1758) }\end{array}$ & 2 & 0,5 & 1 & 0,3 & 1 & 0,3 & - & - & 4 & 1,1 \\
\hline $\begin{array}{l}\text { Rongeur Muridé/ } \\
\text { Arvicolidé }\end{array}$ & & 1 & 0,06 & - & - & - & - & - & - & 1 & 0,06 \\
\hline $\begin{array}{l}\text { Petit mammifère } \\
\text { taille Souris }\end{array}$ & & - & - & - & - & - & - & 1 & 0,07 & 1 & 0,07 \\
\hline Total déterminés & & 46 & 867,1 & 192 & 4271,3 & 206 & 1622,4 & 106 & 1754,7 & 550 & 8515,4 \\
\hline Total indéterminés & & 36 & 113,7 & 138 & 427,3 & 166 & 383,6 & 58 & 106,2 & 398 & 1030,8 \\
\hline Total Mammifères & & 82 & 980,8 & 330 & 4698,6 & 372 & 2006,0 & 164 & 1860,9 & 948 & 9546,2 \\
\hline Oiseaux & & - & - & - & - & - & - & - & - & - & - \\
\hline Oie domestique & Anser cf. domesticus & - & - & 11 & 21,2 & 9 & 13 & 2 & 2,5 & 22 & 36,7 \\
\hline Oie sauvage & Anser sp. & - & - & 1 & 4,3 & 2 & 4,9 & - & - & 3 & 9,2 \\
\hline Canards & Anatinae & 1 & 0,8 & 4 & 1,7 & 7 & 5,3 & 2 & 4,1 & 14 & 11,9 \\
\hline Poule & $\begin{array}{l}\text { Gallus gallus } \\
\text { (Linnaeus, 1758) }\end{array}$ & 5 & 3,2 & 18 & 16,8 & 75 & 64 & 22 & 17,8 & 120 & 101,8 \\
\hline Faisan & $\begin{array}{l}\text { Phasianus colchicus } \\
\text { Linnaeus, } 1758\end{array}$ & - & - & - & - & 1 & 0,6 & - & - & 1 & 0,6 \\
\hline Perdrix rouge & $\begin{array}{l}\text { Alectoris rufa (Linnaeus, } \\
\text { 1758) }\end{array}$ & 1 & 0,3 & - & - & 1 & 0,6 & - & - & 2 & 0,9 \\
\hline Perdrix grise & $\begin{array}{l}\text { Perdix perdix (Linnaeus, } \\
\text { 1758) }\end{array}$ & - & - & - & - & 2 & 0,9 & - & - & 2 & 0,9 \\
\hline $\begin{array}{l}\text { Bécasse des } \\
\text { bois }\end{array}$ & $\begin{array}{l}\text { Scolopax rusticola } \\
\text { Linnaeus, } 1758\end{array}$ & 1 & 0,4 & 1 & 0,3 & 12 & 3,6 & 5 & 1,3 & 19 & 5,6 \\
\hline Courlis cendré & $\begin{array}{r}\text { Numenius arquata } \\
\text { (Linnaeus, 1758) }\end{array}$ & - & - & - & - & - & - & 1 & 0,3 & 1 & 0,3 \\
\hline Pigeons & Columba sp. & 1 & 0,3 & 3 & 2,8 & 4 & 2,3 & 6 & 1,7 & 14 & 7,1 \\
\hline Goeland argenté & $\begin{array}{l}\text { Larus argentatus } \\
\text { Pontoppidan, } 1763\end{array}$ & - & - & 1 & 1,0 & - & - & - & - & 1 & 1,0 \\
\hline $\begin{array}{l}\text { Autour des } \\
\text { Palombes }\end{array}$ & $\begin{array}{l}\text { Accipiter gentilis } \\
\quad \text { (Linnaeus, 1758) }\end{array}$ & - & - & 5 & 11,7 & - & - & - & - & 5 & 11,7 \\
\hline $\begin{array}{l}\text { Faucon } \\
\text { crécerelle }\end{array}$ & $\begin{array}{l}\text { Falco tinnunculus } \\
\text { Linnaeus, } 1758\end{array}$ & - & - & - & - & 2 & 0,6 & - & - & 2 & 0,6 \\
\hline Chouette effraie & Tyto alba (Scopoli, 1769) & - & - & - & - & - & - & 1 & 0,7 & 1 & 0,7 \\
\hline Corneille noire & $\begin{array}{l}\text { Corvus corone Linnaeus, } \\
1758\end{array}$ & - & - & - & - & 1 & 0,7 & - & - & 1 & 0,7 \\
\hline Pie bavarde (?) & $\begin{array}{l}\text { Pica pica } \\
\quad \text { (Linnaeus, 1758) }\end{array}$ & - & - & 2 & 0,8 & - & - & - & - & 2 & 0,8 \\
\hline Total déterminés & & 9 & 5,0 & 46 & 60,6 & 116 & 96,5 & 39 & 28,4 & 210 & 190,5 \\
\hline Total indéterminés & & 3 & 1,0 & 11 & 3,5 & 40 & 6,7 & 32 & 5,3 & 86 & 16,5 \\
\hline Total Oiseaux & & 12 & 6,0 & 57 & 64,1 & 156 & 103,2 & 71 & 33,7 & 296 & 207,0 \\
\hline «Poissons» & & - & - & - & - & - & - & - & - & - & - \\
\hline Raies et requins & Chondrychtyens & - & - & 2 & 0,7 & - & - & 5 & 1,9 & 7 & 2,6 \\
\hline Congre & $\begin{array}{l}\text { Conger conger } \\
\quad(\text { Linnaeus, } 1758)\end{array}$ & 3 & 1,1 & 1 & 0,6 & - & - & 1 & 0,5 & 5 & 2,2 \\
\hline Cyprinidés & Cyprinidae & - & - & - & - & 2 & 0,6 & - & - & 2 & 0,6 \\
\hline Merlu & $\begin{array}{l}\text { Merluccius merluccius } \\
\text { (Linnaeus, } 1758)\end{array}$ & 14 & 14,2 & 43 & 56,6 & 17 & 21,7 & 2 & 1,1 & 76 & 93,6 \\
\hline
\end{tabular}


Table 1. - Suite

\begin{tabular}{|c|c|c|c|c|c|c|c|c|c|c|c|}
\hline \multirow[b]{2}{*}{ Nom vernaculaire } & \multirow[b]{2}{*}{ Nomenclature linnéenne } & \multicolumn{2}{|c|}{ État 5 - XIIIe } & \multicolumn{2}{|c|}{ État 6 - XIIIe-XIVe } & \multicolumn{2}{|c|}{ État $7-$ XIIIe-XIVe } & \multicolumn{2}{|c|}{ État 8 - XIVe } & \multicolumn{2}{|c|}{ Total - XIIIe-XIVe } \\
\hline & & NR & MR & NR & $\mathrm{MR}$ & NR & MR & NR & MR & NR & MR \\
\hline Lieu jaune & $\begin{array}{l}\text { Pollachius pollachius } \\
\text { (Linnaeus, 1758) }\end{array}$ & 1 & 0,9 & 1 & 0,9 & 3 & 3,9 & 1 & 0,7 & 6 & 6,4 \\
\hline Bar & $\begin{array}{l}\text { Dicentrarchus labrax } \\
\text { Linnaeus, } 1758\end{array}$ & - & - & - & - & 3 & 1,4 & 2 & 2,6 & 5 & 4 \\
\hline Dorades & Sparidae & 10 & 24,7 & 15 & 11,6 & 11 & 7,3 & - & - & 36 & 43,6 \\
\hline Grondins & Triglidae & - & - & 1 & 0,3 & 11 & 3,8 & - & - & 12 & 4,1 \\
\hline Pleuronectidés & Pleuronectidae & 2 & 0,9 & 1 & 0,2 & 1 & 0,5 & - & - & 4 & 1,6 \\
\hline Turbot & $\begin{array}{l}\text { Scophthalmus maximus } \\
\text { (Linnaeus, 1758) }\end{array}$ & 1 & 0,5 & 2 & 3,2 & 1 & 0,6 & 1 & 0,2 & 5 & 4,5 \\
\hline Total déterminés & & 31 & 42,3 & 66 & 74,1 & 49 & 39,8 & 12 & 7,0 & 158 & 163,2 \\
\hline Total indéterminés & & 23 & 3,7 & 82 & 11,8 & 43 & 4,2 & 15 & 1,5 & 163 & 21,2 \\
\hline Total «Poissons» & & 54 & 46,0 & 148 & 85,9 & 92 & 44,0 & 27 & 8,5 & 321 & 184,4 \\
\hline
\end{tabular}

à première vue quelque peu contradictoire avec l'histoire du site. Depuis le XIIIe siècle en effet, les puissants vicomtes de Thouars, devenus seigneurs de Talmont, n'occupent plus qu'occasionnellement l'ancienne et prestigieuse forteresse des comtes-ducs d'Aquitaine. Des officiers et une garnison habitent désormais le château.

Dans le cas du cerf, la répartition anatomique des restes est singulière (Fig. 4). Il s'agit presque exclusivement d'éléments du membre pelvien, ce qui n'est pas sans évoquer la découpe ritualisée de la chasse à force, décrite de manière théorique par les traités de vénerie de la fin du Moyen Âge (e.g. Beck et al. 2006; Bord \& Mugg 2008). Le type de rejet découvert ici correspond assez certainement à des déchets de découpe de gros ou demi-gros. L'analyse des documents de la pratique et des comptes vient éclairer l'origine de ce lot tardo-médiéval. Dans ces sources, l'hypothèse d'un atelier de découpe de venaison semble en effet confirmée par la présence d'un personnel seigneurial - attesté en l'absence ou en la présence des vicomtes - missionné spécifiquement pour la chasse à courre et les expéditions de viande depuis le château de Talmont vers celui de Thouars, siège du pouvoir des vicomtes.

Ces différents éléments laissent ainsi penser que le château de Talmont aurait eu, à la fin du Moyen Âge, un rôle de résidence de chasse. La concentration de restes de faune sauvage peut alors être interprétée comme une accumulation due à la préparation sur place de pièces de viande destinées à la table d'autres châteaux détenus par les seigneurs de Talmont, plutôt que le reflet d'une consommation alimentaire in situ. L'archéologie et les textes s'accorderaient ainsi à mettre en évidence une spécialisation fonctionnelle de ce lieu dédié à la production et à l'expédition des produits du monde sauvage pour la table seigneuriale.

Les poissons, une catégorie sous-évaluée par l'archéozoologie? De l'importance du tamisage

Les poissons, s'ils sont bien mentionnés par les comptes, sont parfois absents des études archéozoologiques pour des raisons méthodologiques. Une première remarque s'impose en effet lorsqu'il s'agit de parler de poissons archéologiques: globalement, du fait d'une construction squelettique particulière notamment au niveau de la tête, les poissons, même de grande taille, présentent des os de format moindre que ceux

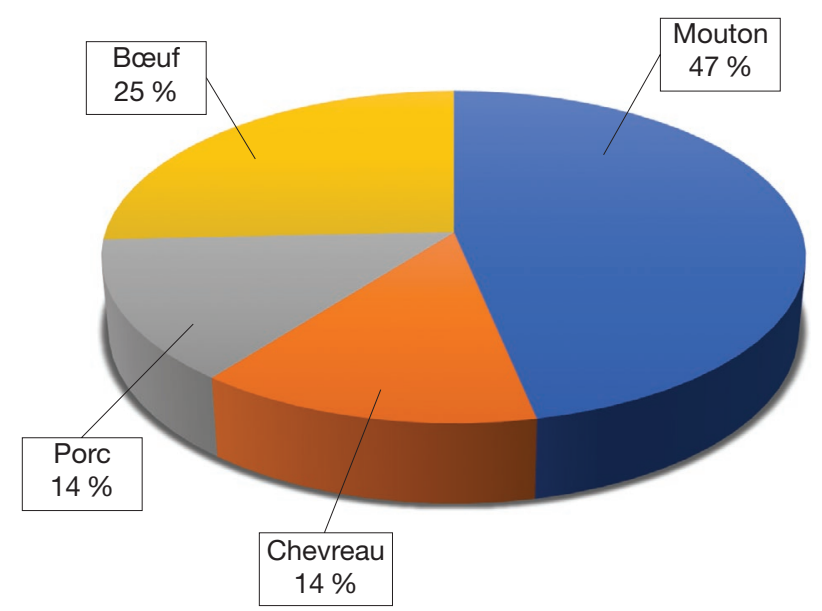

FIG. 3. - Répartition des dépenses en viande au château de Thouars, 22 mai18 août 1440 (étude C. Viaut).

des mammifères et des oiseaux. Cette information vient souligner l'importance des protocoles de prélèvements utilisés pour récolter ces petits vestiges fauniques sur les sites archéologiques. En effet, bien que ce processus soit souvent long et fastidieux, sans tamisage des sédiments, seuls quelques pourcents des os de poissons sont récoltés "à vue» (e.g. pour des exemples chiffrés: Clavel 2001; Borvon 2012). C'est ainsi tout un pan de la faune qui peut faire défaut si de telles méthodes ne sont pas employées. À Talmont, des prélèvements de sédiments ont été réalisés dans certains niveaux. Lavés et tamisés à l'eau sur une maille de $2,8 \mathrm{~mm}$, les restes ichthyologiques en ont été sortis. L'étude de ces petits vestiges a ainsi permis de délivrer de nombreuses informations quant aux poissons consommés; et la liste est relativement longue, avec une quinzaine de taxons différents identifiés pour les niveaux des XIIIe-XIVe siècle.

\section{DE LA RELATIVITÉ DES DISTINCTIONS ENTRE ANIMAUX Lus et becquet, anguille et anguillette}

Au-delà des approches quantitatives de la représentation et du nombre des animaux, l'approche qualitative des espèces animales en contexte castral passe, en archéozoologie comme dans l'étude des archives comptables, par l'établissement de listes d'espèces qui permettent une approche de la diversité 
et la hiérarchie des animaux consommés. Si l'établissement des listes de faune passe par la détermination des ossements en archéozoologie, l'identification des espèces à partir des archives s'appuie sur la perception qu'avaient les scribes médiévaux des différents animaux achetés puis consommés. L'établissement de ces listes à partir des archives médiévales de Talmont et de Thouars a notamment permis de mettre en évidence, parmi les nombreuses appellations vernaculaires relevées, des distinctions ne relevant pas de l'espèce au sens moderne du terme, mais d'autres catégories porteuses de sens aux yeux des acheteurs et consommateurs de ces animaux.

Les comptes mettent notamment l'accent, à travers le vocabulaire employé, sur un critère de distinction rarement mis en valeur dans les études archéozoologiques : leur format. Les achats de brochets (lus) (Esox lucius L., 1758) dans les comptes de Thouars sont par exemple clairement distingués des achats de becquets. Les dictionnaires d'ancien et moyen français (Godefroy 1898), s'ils notent l'identité $\mathrm{du}$ becquet et du brochet, ne permettent pas de saisir dans son contexte la différence tracée par les consommateurs médiévaux entre deux animaux appartenant pour nous à la même espèce. Il s'agit essentiellement d'une distinction liée à l'âge, et donc à la taille, qui se traduit par une plus grande valeur accordée aux vieux brochets. La documentation comptable ne permet pas, par ailleurs, de distinguer des usages différenciés des brochets selon leur taille en cuisine. Les distinctions de format entre individus de la même espèce peuvent, dans certains cas, être porteuses de significations d'ordre économique et social.

Ainsi, les comptes thouarsais n'enregistrent pas seulement les dépenses pour la table seigneuriale, mais également celles occasionnées par la nourriture due aux travailleurs accomplissant transports et travaux au château. Le 17 septembre 1440, les comptes notent la dépense engagée pour la nourriture d'autres bouviers apportant du bois de construction depuis le parc seigneurial:

\section{Led. jour pour ung cincquante d'anguilletes pour bailler a} bovers qui amenoient nos bois ....................3 s. $2 \mathrm{~d}$. (Archives nationales: 1AP/5561, premier cahier, fo $18 \mathrm{v}^{\circ}$ )

Le 21 juin 1441, des anguillettes, ou petites anguilles (Anguilla anguilla (L., 1758)), sont achetées à nouveau pour nourrir des bouviers:

Item ledit jour pour trois XIInes d'anguillectes pour diz huit boviers qui admenerent neuf charretees $d u$ pré du Sault presTexme lequel avoit esté achapté pour ce ..............2s. $6 \mathrm{~d}$. (Archives nationales: $1 \mathrm{AP} / 5561$, premier cahier, fo $113 \mathrm{r}^{\circ}$ )

D'autres sont encore achetées le surlendemain:

Item pour une cincquantaine d'anguillecte despencees par neuf hommes qui estoient a la grange pour antasser fains et pour XIX boviers qui amenerent XI charretz de fain du pré du Sault et du pré Jehan de Doulces ................. 3s. $3 d$. (Archives nationales: 1AP/5561, premier cahier, fo113vo)
Nous pourrions ainsi multiplier les exemples, tant les comptes donnent à voir les anguillettes comme une nourriture destinée aux travailleurs de force, réservée à la population laborieuse, aux domestiques du château. L'anguille est fréquente dans les comptes: on en achète à 80 reprises lors du Carême de 1441 au château de Thouars, et il s'agit d'un poisson couramment consommé à la fin du Moyen Âge, quoique de moins en moins, en faveur notamment des poissons marins (Clavel 2001, 2013; Clavel \& Cloquier 2015). Elle est ici clairement distinguée de l'anguillette non seulement par son nom, mais aussi par sa destination. Jamais mentionnée aussi précisément que pour sa version juvénile, il $\mathrm{y}$ a fort à parier que l'anguille adulte finisse sa route sur la table seigneuriale ou, tout du moins, sur celle des officiers et familiers seigneuriaux du château de Thouars. Ce critère du format, dont on voit pourtant l'éminente portée sociale en termes d'alimentation, est pourtant peu souvent mis en valeur dans les études archéoichthyologiques (pour un des rares exemples connus, cf. Clavel 2001: 185 [poissons plats]). Ici, les catégorisations médiévales s'écartent considérablement des nôtres en vertu des critères employés pour distinguer les animaux les uns des autres dans le contexte social de leur consommation.

\section{De la difficulté de distinguer sauvage et domestique en archéozoologie: entre cochon et sanglier...}

Une question épineuse en archéozoologie réside dans la distinction entre le porc (Sus scrofa f. domesticus Erxleben, 1777) et le sanglier (Sus scrofa scrofa L., 1758). Ils font en effet partie de la même espèce, le premier étant une forme domestique du second (Larson et al. 2005, 2007). La distinction ostéologique entre ces deux formes n'est alors pas toujours aisée. Celle-ci est en effet compliquée par le fort polymorphisme des deux sousespèces et par leur interfécondité (hybridation). L'analyse de différents critères morphologiques et ostéométriques, réalisée lors d'une étude portant sur plus de 4000 restes de suidés datés du XIe siècle, permet de dire que la distinction de ces deux formes n'est possible que sur des critères de taille (Borvon 2012), suivant en cela l'analyse de C. Becker (1980) réalisée sur le corpus conséquent d'Haithabu (Allemagne, IXe-XIe siècle). Seuls les ossements les plus grands sont alors attribués au sanglier. Des mensurations proches de ceux-ci soulèvent néanmoins toujours la question de la présence d'individus de petite taille, ou inversement, de porcs de grande taille, ou bien encore d'éventuels hybrides. Du fait de cette identification reposant sur le format des ossements, il est très probable que la présence de cette espèce soit quelque peu sous-estimée. À Talmont, la taille importante de certains ossements permet sans conteste de témoigner de la présence du sanglier, et ce toutes périodes chronologiques confondues. Si la difficulté est récurrente en archéozoologie, la distinction de la forme domestique et de la forme sauvage ne pose en revanche aucun problème dans les textes, puisque des termes différents sont employés pour qualifier cochon et sanglier (Delort 1990).

\section{... et des espèces non distinguées dans les textes}

Les documents de la pratique ne sont souvent d'aucune aide pour distinguer des espèces que nous considérons à l'heure actuelle comme différentes, par exemple parmi les 


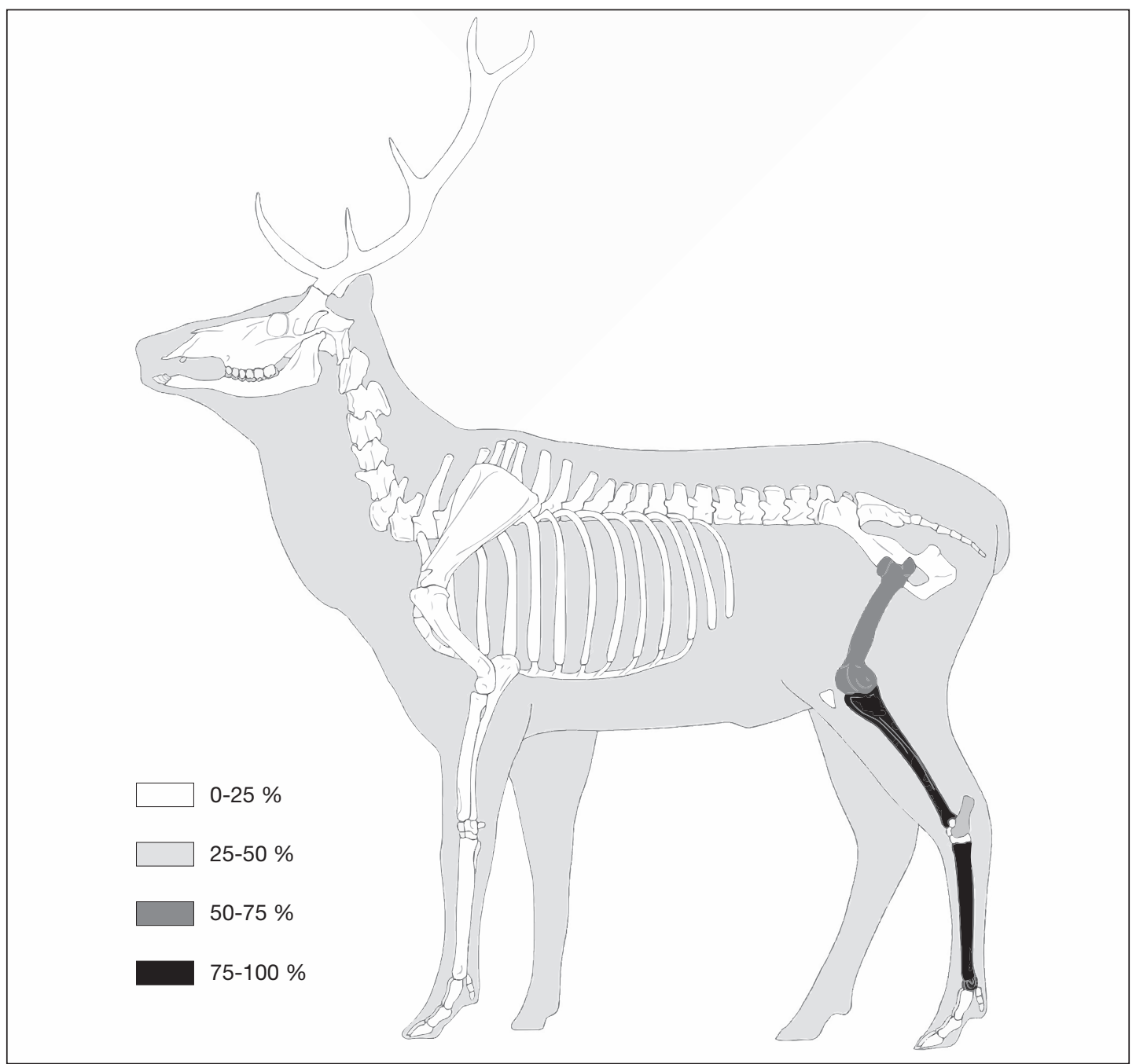

FIG. 4. - Répartition relative en taux pondéré de présence (TPp) (Rodet-Belarbi et al. 2002)-des organes squelettiques de cerf (US 1226, état 9: fin XIVe-XVe siècle, secteur 1 : bâtiment nord; Borvon \& Viaut 2018).

différents poissons plats, ou bien encore parmi les canards sauvages, peu représentés dans les sources écrites. Ces derniers sont divers à Talmont, avec la présence du très classique canard colvert (Anas platyrhynchos L., 1758), mais aussi des deux espèces de sarcelles (Anatidae), des possibles canards souchet (Spatula clypeata L., 1758), siffleur (Anas penelope L., 1758), pilet (Anas acuta L., 1758), ou bien encore des fuligules (Aythya Boie, 1822) et macreuses (Melanitta Boie, 1822) (Woelfle 1967; Collections du Muséum national d'Histoire naturelle). Nous pouvons ainsi émettre l'hypothèse que certaines distinctions établies dans la littérature archéozoologique sur la base des espèces actuellement définies n'ont pas réellement eu d'importance aux yeux des acheteurs et des consommateurs médiévaux. La définition précise de ces espèces est cependant d'un grand intérêt en termes d'analyse des milieux naturels et agricoles dans lesquels s'insèrent les sites archéologiques. L'analyse environnementale ainsi réalisée à partir des vestiges conservés au château de Talmont illustre cet intérêt (Borvon 2017b). En effet, en plus des canards mentionnés qui fréquentent des étendues aquatiques diverses (d'eau douce à marines, en passant par les eaux saumâtres suivant les espèces), la présence du courlis cendré (Numenius arquata (L., 1758)) témoigne de la présence de prairies côtières en général humides; celle de la bécasse de bois (Scolopax rusticola L., 1758), de forêts entrecoupées de champs et de clairières; et celle des perdrix (Alectoris rufa (L., 1758) et Perdix perdix (L., 1758)) de milieux plutôt ouverts, tels les terres cultivées et friches, pâturages, landes ou bien encore dunes (Peterson et al. 2006; Mullarney et al. 2008).

LES INCONNUS RESPECTIFS. QUAND DES SOURCES DIFFÉRENTES N'IGNORENT PAS LES MÊMES ESPÈCES

Au-delà même des différences manifestes d'appellation des espèces animales se pose la question de la présence ou de l'absence de certaines dans les listes de faune établies respectivement à partir des assemblages fauniques et de la documentation comptable. L'exemple du site de Talmont au XVe siècle est à ce titre particulièrement révélateur, en ce qu'il donne à voir un véritable fossé entre la diversité de la consommation 


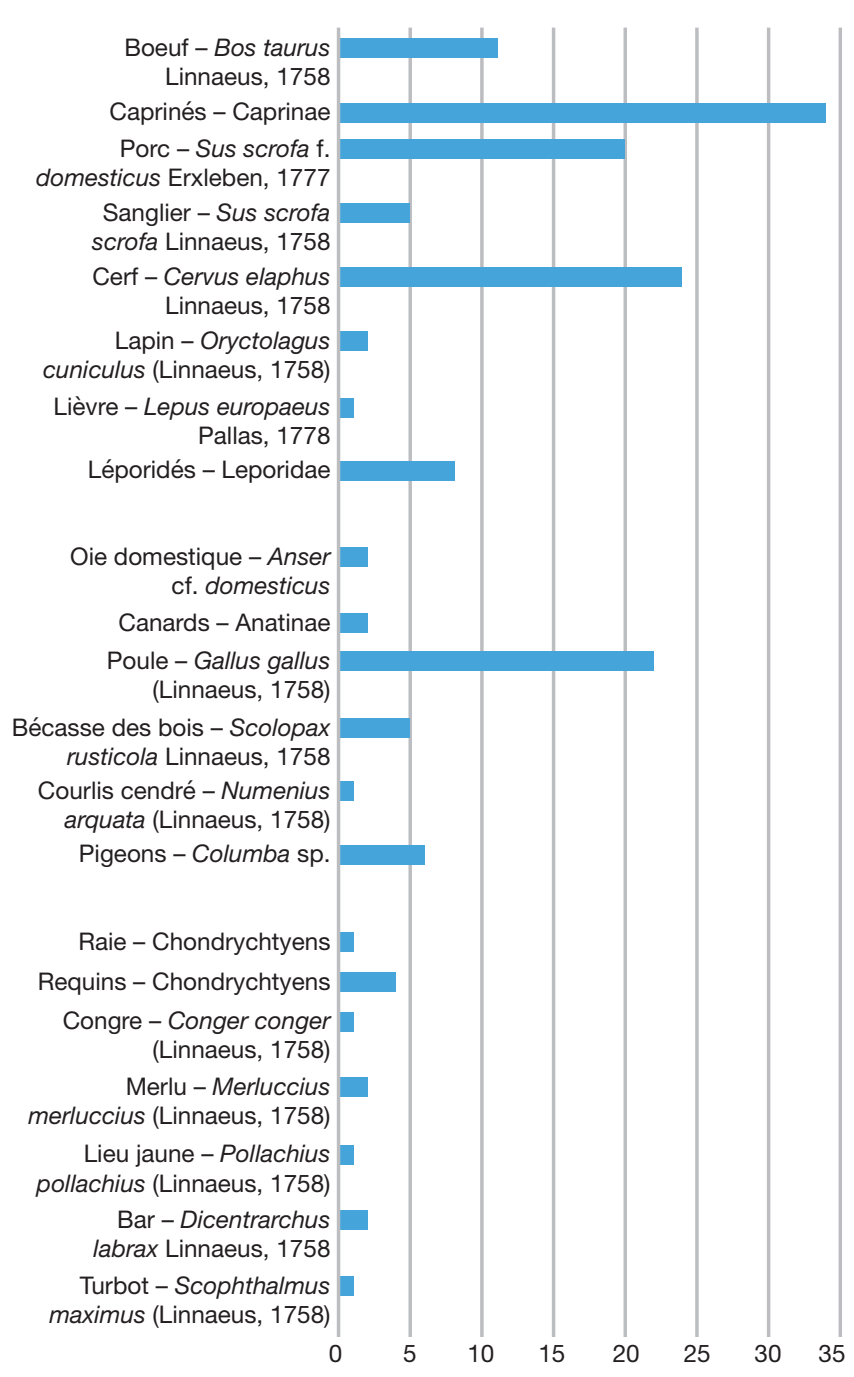

FIG. 5. - Nombre de restes (NR) par espèce (NR déterminés total = 155) Talmont, état 8 (XVe siècle, secteur 1: bâtiment nord; Borvon 2017b).

carnée révélée par l'archéologie d'une part, et celle des textes d'autre part. L'état 8 de la grande salle nord du château de Talmont, qui correspond à une occupation de la fin du XIVe_ début du XVe siècle, a en effet permis d'identifier les restes d'espèces variées (Fig. 5).

Les comptabilités contemporaines de cet état d'occupation du bâtiment nord sont loin de montrer une telle diversité (Fig. 6). Le relevé des espèces animales consommées par le vicomte de Thouars lors de sa visite à Talmont en septembre 1446 (Archives nationales: 1AP/557), ne montre ainsi qu'une diversité très réduite.

Cette différence tient à la nature fondamentalement différente de la constitution des deux types de sources. Alors que les textes, comme dans ce dernier cas, n'enregistrent des achats que sur un temps très court - quelques jours -, les niveaux d'occupation archéologiques piègent des restes sur des durées beaucoup plus importantes. Le huitième état d'occupation du bâtiment nord du château de Talmont pourrait ainsi correspondre à une occupation longue de plusieurs années à plusieurs décennies (Béthus 2016). Dans le

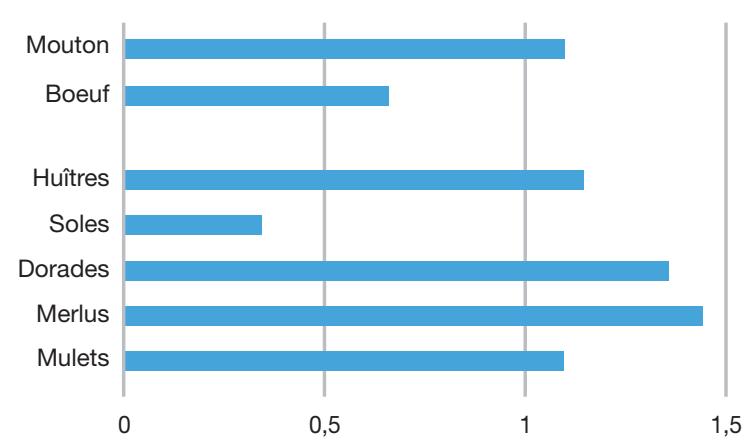

FIG. 6. - Sommes dépensées en livres tournois (I. t.) pour les achats de viande, Talmont, septembre 1446 (étude C. Viaut).

cas de sites comme Talmont, où les comptes ne renseignent que de courtes périodes, à la différence de Thouars, l'usage exclusif des sources écrites nous mènerait à ignorer la présence de très nombreuses espèces animales. Cependant, même lorsque les sources écrites comptables renseignent la consommation sur une durée plus longue, plusieurs mois ou plusieurs années, la plus grande partie des espèces sauvages en sont quasi-absentes.

Si la plupart des espèces consommées sont présentes à la fois dans les textes et dans les niveaux archéologiques, tel n'est pas le cas de nombreux taxons non consommés mais retrouvés dans les niveaux archéologiques, et notamment les espèces familières et commensales. Les chiens (Canis lupus familiaris (L., 1758)) ne sont évoqués que parce qu'il s'agit principalement de chiens de chasse, très secondairement de compagnie, mais les chats (Felis catus (L., 1758)) n'apparaissent pour ainsi dire jamais, pour ne rien dire des rongeurs. Une seule mention écrite en est faite à Talmont, concernant les réparations du grenier et le déplacement des grains en raison des dégâts causés par les rats (Archives départementales de la Vendée: 1E/1245, fo26ro). Les sources archéozoologiques en revanche, attestent bien de la présence du rat noir (Rattus rattus (L., 1758))(Borvon 2016), espèce probablement surreprésentée au détriment d'espèces plus petites (souris [Mus musculus L., 1758], mulots [Apodemus Kaup, 1829], musaraignes [Soricinae] et autres) du fait de la maille de tamis employée.

Les comptes doivent ainsi être compris dans le sens d'un panel d'espèces élevées ou pêchées principalement dans le seul but de la consommation et ne concernent que peu les autres animaux. Ici, ce ne sont pas tant les différences de catégorisation de ces animaux qui font écran à leur connaissance dans les enceintes castrales, mais plutôt la nature du document - comptes des dépenses de bouche - très centrée sur l'alimentation, tandis que l'archéozoologie n'opère pas de tri préalable dans l'utilisation possible des espèces par l'homme lors de l'établissement de la liste de ces mêmes espèces à partir des restes osseux. Certaines catégories animales renseignées par l'une et l'autre source permettent parfois de comparer entre elles les informations apportées par les différentes fabriques documentaires, particulièrement dans le cas des oiseaux de proie. 
POUR UNE APPROCHE INTERDISCIPLINAIRE DES CATÉGORIES ANIMALES EN CONTEXTE CASTRAL: LE CAS DES OISEAUX DE PROIE Des animaux assez bien représentés dans les textes...

Les rapaces sont relativement peu évoqués comparativement aux espèces consommées dans les textes médiévaux relatifs aux châteaux de Talmont et Thouars, mais le sont tout de même assez régulièrement. À la fin du XIIIe siècle, le Livre rouge de Thouars (Archives nationales: 1AP/2258), censier et registre féodal des possessions des vicomtes de Thouars, précise la manière dont les oyseaus de rapt entrent en possession des seigneurs de Talmont : ils sont capturés en forêt de Jard et font l'objet d'une redevance seigneuriale (Archives nationales: $1 \mathrm{AP} / 2258$, fo $\left.17 \mathrm{r}^{\circ}\right)$. Deux siècles et demi plus tard, les comptes des recettes et des mises de la seigneurie de Talmont font état de ventes de faucons toujours acquis par le biais des redevances seigneuriales (Archives départementales de Vendée: 1E/1249, fo $25 \mathrm{r}^{\circ}$ ). Les oiseaux de proie sont désignés par un vocabulaire spécifique: faucon gentis (faucon pélerin, Falco peregrinus Tunstall, 1771), lener (faucon lanier, Falco biarmicus Temminck, 1825, tiercelet gentis (tiercelet pélerin). Ces mots désignent des catégories spécifiques d'oiseaux de proie dans un vocabulaire propre à la fauconnerie, selon l'espèce ou le sexe (Van den Abeele 1990; Paravicini Bagliani \& Van den Abeele 2000).

\section{... mais plutôt rares sur les sites archéologiques}

Généralement ces oiseaux sont assez peu représentés sur les sites médiévaux (e.g. Clavel 2001; Borvon 2012) et le site de Talmont ne fait pas exception. Cinq ossements d'autour des palombes Accipiter gentilis (L., 1758) (Fig. 7) sont néanmoins à signaler dans des niveaux des XIIIe-XIVe siècles. Appartenant probablement tous à un même individu adulte, il s'agit certainement une femelle puisque chez cette espèce celles-ci sont bien plus grandes et massives que les mâles (Peterson et al. 2006). Il s'agit d'un oiseau couramment utilisé pour la chasse dite de bas vol ou autourserie (Benoist 1980 ; Chenu \& des Murs 2007; Bord \& Mugg 2008). Celle-ci correspond à des rapaces qui chassent en poursuite à partir du poing, contrairement aux faucons qui chassent en piqué (chasse dite de haut vol ou fauconnerie, devenue ensuite générique pour parler de chasse au vol). Dans la nature, cet oiseau fréquente plutôt les massifs forestiers (Peterson et al. 2006), ce qui fait qu'affaité, il est efficace même en terrain peu dégagé. La plupart des ouvrages, anciens comme actuels, le désignent comme le plus efficace des oiseaux de chasse dans nos régions (Chenu \& des Murs 2007; Bord \& Mugg 2008). À Talmont il est ainsi fort possible que la femelle identifiée soit détenue en captivité en vue d'une activité de chasse. Signalons que pour la période qui nous intéresse, ces oiseaux sont forcément prélevés dans la nature puisqu'il n'y a pas de reproduction en captivité; d'ailleurs, avant le XIII siècle, les traités de chasse n'intéressent que les rapaces. Ils exposent les difficultés d'affaitage, de garde et d'entretien en captivité ainsi que les maladies (Benoist 1980; Van den Abeele 1990; Lagae 2005; Chenu \& des Murs 2007). De telles difficultés au maintien en captivité sont probablement mises en évidence à Château Ganne (Calvados, seconde moitié du XIe-milieu XIIIe siècle) : les déformations observées sur le

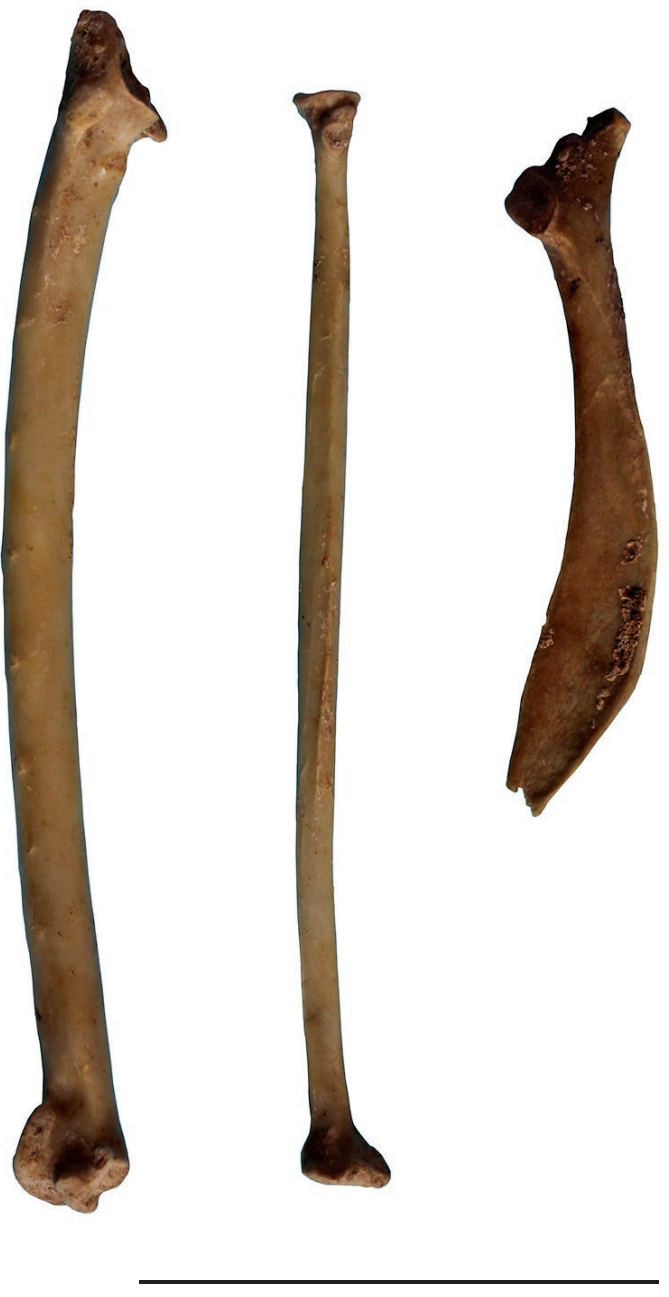

FIG. 7. - Ulna, radius et scapula d'autour des palombes Accipiter gentilis (Linnaeus, 1758), château de Talmont (état 6 , XIIIe-XIVe siècles, secteur 1: bâtiment nord; cliché A. Borvon).

squelette d'un autour témoignent probablement de la difficulté à faire grandir correctement un oiseau prélevé dans la nature (Borvon \& Flambard Héricher 2014).

\section{CONCLUSION}

La fauconnerie est-elle un exemple de la différence entre cladistique contemporaine et conceptions techniques médiévales ? Les raccords difficiles entre la nomenclature contemporaine et le vocabulaire médiéval des oiseaux de proie illustrent le fond du problème que nous avons tenté de définir ici. Alors que les méthodes de l'archéozoologie se font toujours plus pointues dans l'identification des espèces, la détermination de l'âge et du sexe des individus, il faut garder en mémoire que certaines distinctions importantes à nos yeux, telles les différences entre les espèces de canards sauvages, n'avaient pas lieu d'être pour les occupants des sites que nous étudions. D’un autre côté, certaines distinctions ne prenant pas en compte l'espèce, mais le format des individus par exemple, établissent une séparation qui n'est 
pas particulièrement mise en avant en archéozoologie, et qui pourtant est lourde de sens sur le plan social et culturel, comme l'a singulièrement montré l'exemple des anguilles et des anguillettes consommées au château de Thouars. Il serait ainsi très intéressant de relire les résultats des investigations archéozoologiques à la lumière des catégories porteuses de sens à l'époque considérée. À l'image de ce premier débroussaillage méthodologique, mené conjointement par un historien-archéologue et une archéozoologue, un tel travail d'interprétation ne pourra s'effectuer que dans un cadre interdisciplinaire, associant analyse des vestiges osseux et étude du statut social, analyse des représentations et des conditions de consommation des espèces identifiées.

\section{Remerciements}

Nous tenons à remercier pour leur aide précieuse: Teddy Béthus, archéologue médiéviste au département de la Vendée, responsable d'opération des fouilles programmées du Château de Talmont; Nicolas Prouteau, maître de conférences en archéologie médiévale à l'Université de Poitiers; tous les fouilleurs du château de Talmont. Que soient aussi remerciés les relecteurs de cet article.

\section{RÉFÉRENCES}

Аввотт F. 2012. - Des comptes d'apothicaires: les épices dans la comptabilité de la Maison de Savoie (XIVe et XVe s.). Cahiers lausannois d'Histoire médiévale (51), 210 p.

Audoin-Rouzeau F. 1986. - Ossements animaux du Moyen Âge au monastère de la Charité-sur-Loire. Éditions de la Sorbonne, Paris, 168 p. (Coll. Histoire ancienne et médiévale).

Audoin-Rouzeau F. 1995. - Compter et mesurer les os animaux. Pour une histoire de l'élevage et de l'alimentation en Europe de l'Antiquité aux Temps Modernes, in Straus A. \& Verley P. (éds), Consommation. Histoire et Mesure 10 (3/4): 277-312.

Beck C., Rodet-Belarbi I. \& Marinval M.-C. 2006. - Cerf et sanglier au Moyen-Âge. Du discours à la Pratique, in SiDÉRA I., Vila E. \& ERIKSON P. (éds), La chasse. Pratiques sociales et symboliques. De Boccard, Paris: 235-243.

BECKER C. 1980. - Untersuchungen an Skelettresten von Haus- und Wild schweinen aus Haithabu. Karl Wachholtz, Neumünster, 94 p. (Coll. Berichteüber die Ausgrabungen in Haithabu; 15).

BENOIST J.-O. 1980. — La chasse au vol. Techniques de chasse et valeur symbolique de la volerie, in CENTRE D'ÉTUDES MÉDIÉVALES (éd.), La chasse au Moyen Âge, Actes du colloque de Nice (22-24 juin 1979). Publications de la Faculté des lettres et des sciences humaines de Nice, Nice: 117-131.

BÉTHus T. 2016. - Le bâtiment nord du château de Talmont SaintHilaire: rapport de fouille programmée pluriannuelle 2013-2015. SRA Pays de la Loire, Nantes, 202 p.

Béthus T. \& Prouteau N. 2014. - Les mutations du castrum de Talmont (Vendée) : naissance et transformations d'une forteresse bas-poitevine (Xe-XIII siècles), in BOURGEOIs L. \& REMY C. (éds), Demeurer, défendre et paraître: orientations récentes de l'archéologie des fortifications et des résidences aristocratiques médiévales entre Loire et Pyrénées. Association des Publications Chauvinoises, Chauvigny: 231-257.

Bord L.-C. \& MugG J.-P. 2008. — La chasse au Moyen Âge. Occident latin, VIe-XVe siècle. Compagnie des éditions de la Lesse; Éditions du Gerfaut, Paris, 356 p.
BORVON A. 2012. - Acquisition des ressources animales, alimentation carnée et distinction sociale en Anjou de la fin du Xe au début du XII siècle. Étude archéozoologique du site de Montsoreau (Maine-etLoire). Thèse de doctorat, Université Paris 1 Panthéon-Sorbonne, 743 p. https://halshs.archives-ouvertes.fr/tel-00669956/, dernière consultation: 30/06/2020.

BORVON A. 2016. - Étude archéozoologique du château de Talmont Saint-Hilaire (Vendée), première approche, in BÉTHUS T. (éd.), Le bâtiment nord du château de Talmont Saint-Hilaire, rapport de fouille triennale 2013-2015. Rapport d'étude spécialiste. SRA Pays de la Loire, Nantes: 95-106.

Borvon A. 2017a. - Étude des restes fauniques du château de Suscinio, in Vincent K. \& DubOIS A. (éds), Le château de Suscinio (Morbihan - Sarzeau), le logis nord. Rapport de fouilles programmées 2013-2015. Volume 1, Tome II. SRA Bretagne, Rennes : 330-401.

BorVON A. 2017b. - Étude des vestiges fauniques du secteur 1, in BÉTHus T. (éd.), Le château de Talmont (Talmont Saint-Hilaire, Vendée), rapport final d'opération 2017. Rapport d'étude spécialiste. SRA Pays de la Loire, Nantes: 69-108.

BORVON A. \& VIAUT C. 2018. — Les vestiges archéozoologiques de l'état 9 (fin XIVe-XVe siècle), in BÉTHUS T. (éd.), Le château de Talmont (Talmont Saint-Hilaire, Vendée), rapport final d'opération 2018. SRA Pays de la Loire, Nantes: 101-120.

Borvon A. \& Flambard Héricher A.-M. 2014. - Aménagement de la cuisine et alimentation carnée au Château Ganne (la Pommeraye, Calvados, XIe-XIVe siècle), in Cocula A.-M. \& Cомвет M. (éds), Châteaux, cuisines et dépendances. Ausonius, Bordeaux: 79-94.

CASSET M. 2017. - Goûts et choix de consommation alimentaires au château de Hambye au début du XVe siècle, in LAURIOUX B. (éd.), De la nature à la table au Moyen Âge: l'acquisition des aliments. CTHS, Paris: 55-66. (Coll. Actes des congrès nationaux des sociétés historiques et scientifiques).

CASSET M. 2018. - La consommation de produits de la mer à la cour du duc de Bretagne pendant son exil en Angleterre (13771378). Anthropozoologica 53 (5): 73-80. https://doi.org/10.5252/ anthropozoologica2018v53a5

Chanson F. 2007. - Les comptes de la seigneurie de Talmont (14121499). Étude économique et sociale du Talmondais à la fin du Moyen Âge. Mémoire de Master 2, Université de La Rochelle, 168 p. CHARBONNIER P. 1971. - L'alimentation d'un seigneur auvergnat au début du XVe siècle, in CONGRÈS NATIONAL DES SOCIÉTÉS SAVANTES. SECTION D'HISTOIRE MODERNE ET CONTEMPORAINE (éd.), L'alimentation et ses problèmes. Actes du quatre-vingt-treizième congrès national des sociétés savantes, Tours, 1968. Bibliothèque nationale, Paris: 77-101.

Chenu J.-C. \& Des Murs O. 2007. — La fauconnerie ancienne et moderne. Hermann, Paris, 176 p.

Clavel B. 2001. - L'animal dans l'alimentation médiévale et moderne en France du Nord (XIIIe-XVIIe siècles). Revue archéologique de Picardie 19 (1), 204 p. https://doi.org/10.3406/pica.2001.3065

Clavel B. 2013. - La pêche et la consommation du poisson en pays d'Aunis (Charente-Maritime) au Bas Moyen Âge et au début de l'époque Moderne: premiers résultats des analyses archéozoologiques, in Daire M.-Y., DUPONT C., BAUdrY A., Billard C., Large J.-M., Lespez L., Normand É. \& SCARre C. (éds), Anciens peuplements littoraux et relations Homme/ Milieu sur les côtes de l'Europe atlantique. BAR International Series 2570: 429-435.

Clavel B. \& Cloquier C. 2015. - La consommation d'animaux aquatiques dans le bassin de la Somme durant les périodes médiévale et moderne, in DemeulenAere-DouYère C. (éd.), Tous à table! Repas et convivialité. CTHS, Paris: 83-98. (Coll. Actes des congrès nationaux des sociétés historiques et scientifiques).

ContAmine P. 2011. - Château, consommation et commercialisation dans la France de la fin du Moyen Âge: que faisait-on des redevances et des prélèvements seigneuriaux en nature? Étude 
de cas, in CAUChIES J.-M. \& GUISSET J. (éds), Lieu de pouvoir, lieu de gestion. Le château aux XIIIe-XVIe siècles: maîtres, terres et sujets. Brepols, Turnhout: 243-258.

DELORT R. 1990. - Quels animaux les seigneurs chassaient-ils dans les forêts à la fin du Moyen Âge?, in CHASTEL A. (éd.), Le Château, la chasse et la forêt. Sud-Ouest, Bordeaux: 165-171.

FOREST V. 1997. — Données biologiques et données zootechniques anciennes. Essai de mise en équivalence. Revue de Médecine Vétérinaire 148 (12): 951-958.

GODEFroY F. 1898. - Dictionnaire de l'ancienne langue française et de tous ses dialectes du IXe au XVe siècles: composé d'après le dépouillement de tous les plus importants documents manuscrits ou imprimés qui se trouvent dans les grandes bibliothèques de la France et de l'Europe. Vieweg, Paris, $792 \mathrm{p}$.

Horard-Herbin M.-P. \& Vigne J.-D. 2005. - Animaux, environnements et sociétés. Errance, Paris, $191 \mathrm{p}$.

JEANNEAU C. 2013. - Émergence et affirmation des familles seigneuriales à la frontière des grandes principautés territoriales: les seigneurs de la Garnache et les vicomtes de Thouars, in AURELL M. (éd.), Le médiéviste et la monographie familiale: sources, méthodes et problématiques. Brepols, Turnhout: 161-187.

LAGAE É. 2005. - Un traité inédit d'autourserie et de fauconnerie en moyen français. Anthropozoologica 40 (2) : 81-98.

Larson G., Albarella U., Dobney K., Rowley-Conwy P., SCHIbler J., Tresset A., Vigne J.-D., Edwards C. J., SChlumbaum A., Dinu A., Balaçsescu A., Dolman G., Tagliacozzo A., Manaseryan N., Miracle P., Van Wijngaarden-Bakker L., Masseti M., Bradley D. G. \& Cooper A. 2007. - Ancient DNA, pig domestication, and the spread of the Neolithic into Europe. Proceedings of the National Academy of Sciences of the United States of America (PNAS) 104 : 15276-15281. https:// doi.org/10.1073/pnas.0703411104

larson G., Dobney K., Albarella U., Fang M., MatisooSmith E., Robins J., Lowden S., Finlayson H., Brand T., Willerslev E., Rowley-CONWy P., ANDERSSON L. \& COOPER A. 2005. - Worlwide phylogeography of wild boar reveals multiple centers of pig domestication. Science 307: 1618-1621. https:// doi.org/10.1126/science.1106927

LAURIOUX B. \& MOIREZ P. 2008. - Pour une approche qualitative des comptes alimentaires: cour de France et cour de Rome à la fin du Moyen Âge. Food and History 4 (1): 45-66. https://doi. org/10.1484/J.FOOD.2.302443

LECOINTRE G. \& LEGUYADER H. 2001. — Classification phylogénétique du vivant. Belin, Paris, $543 \mathrm{p}$.

Morrez P. 2009. - L'approvisionnement de l'Hôtel du Roi de France à la fin du XIVe siècle d'après les sources comptables, in Ravoire F. \& Dietrich A. (éds), La cuisine et la table dans la France de la fin du Moyen Âge: contenus et contenants du XIVe au XVIe siècle. CRAHM, Caen: 79-83.

MOREL Y. 2006. - Les banquets à la cour de Bourgogne au XVe siècle: récits des chroniqueurs et données des comptes. Food and History 4 (1): 67-84 https://doi.org/10.1484/J.FOOD.2.302444

Morel Y. 2008. - Sources comptables et activités quotidiennes: quel est l'apport des écrous de dépenses de l'Hôtel de Bourgogne pour l'histoire de l'alimentation? Questes. Revue pluridisciplinaire d'études médiévales 15: 82-91 https://doi.org/10.4000/questes.3478

Mullarney K., Svensson L., ZetTerström D. \& Grant P. J. 2008. - Le guide ornitho. Les 848 espèces d'Europe. Delachaux \& Niestlé, Paris, 400 p.

NoËL A. 2017. - La consommation de volatiles dans un hôtel aristocratique à la fin de la guerre de Cent Ans, in Laurioux B. (éd.), De la nature à la table au Moyen Âge: l'acquisition des aliments. CTHS, Paris: 67-74. (Coll. Actes des congrès nationaux des sociétés historiques et scientifiques).

Paravicini Bagliani A. \& VAN Den Abeele B. 2000. - La chasse au Moyen Âge: société, traités, symboles. Sismel - Edizioni del Galluzzo, Florence, 266 p.

Peterson R., Mountfort G., Hollom P. A. D. \& GérouDET P. 2006. - Guide Peterson des oiseaux de France et d'Europe. Delachaux \& Niestlé, Paris, 534 p.

Rodet-Belarbi I., Olive C. \& Forest V. 2002 - Dépôts archéologiques de pieds de mouton et de chèvre: s'agit-il toujours d'un artisanat de la peau?, in AUDOIN-ROUZEAU F. \& BEYRIES S. (éds), Le travail du cuir de la Préhistoire à nos jours. APDCA, Antibes: 315-349.

SAmaran C. \& Courteault H. 1930. - Le chartrier des La Trémoille. Archives nationales, Paris, 43 p.

VAN DEN ABEELE B. 1990. — La Fauconnerie dans les lettres françaises du XIIe au XIVe siècle. Leuven University Press, Louvain, 348 p.

VIAUT C. 2018a. - Du lieu de pouvoir à l'espace vécu: le château de Talmont du XIIIe au XVe siècle. Thèse pour le diplôme d'archiviste paléographe, École nationale des Chartes, Paris, $617 \mathrm{p}$

VIAUT C. 2018b. - Du lieu de pouvoir à l'espace vécu: le château de Talmont du XIIIe au XVe siècle. Positions des thèses soutenues par les élèves de la promotion 2018 pour obtenir le diplôme d'archiviste paléographe. Ecole nationale des Chartes, Paris: 169-179.

VINCENT K. 2017. - Le château de Suscinio (Morbihan-Sarzeau). Le logis nord. Tome 1, Synthèse et illustrations. Rapport de fouille programmée 2013-2015. SRA Bretagne, Rennes, 3 volumes.

VinCENT K., DubOIS A. \& Borvon A. 2018. — Les parcs du château de Suscinio, résidence des ducs de Bretagne (Morbihan, France), in Ettel P., Flambard Héricher A.-M. \& O'Conor K. D. (éds), Château Gaillard: l'environnement du château. Actes du colloque international de Roscommon (Irlande, 14-18 août 2016). Presses universitaires de Caen, Caen: 283-294. (Coll. Château Gaillard. Études de castellologie médiévale; 28).

VISSIÈRE L. 2010. - Des archives et des armes: la renaissance thouarsaise des La Trémoïlle au XVe siècle, in CONTAMINE P. \& VISSIÈRE L. (éds), Les chartriers seigneuriaux: défendre ses droits, construire sa mémoire, XIIIe-XXIe siècle. Société de l'histoire de France, Paris: 191-208.

WOELFLE E. 1967 - Vergleichend morphologische Untersuchungen an Einzelknochen des postcranialen Skelettes in Mitteleuropa vorkommen der Enten, Halbgänseund Säger. Inaugural-Dissertation zur Erlangung der tiermedizinischen Doktorwürde der Tierärztlichen Fakultät, Ludwig-Maximilians-Universität München, 256 p.

WoOlgar C. M. 1992. - Household Accounts from Medieval England. Oxford University Press, 2 vols, $486+396$ p. 